\title{
Cancer is a word, not what defines you: Women with breast cancer
}

\author{
Rekha S G ${ }^{1 *}$, Sanjay M Peerapur ${ }^{2}$, Aaron Roy $^{3}$, Tejaswini B H ${ }^{4}$ \\ ${ }^{1}$ Nurse Specialist, ${ }^{2}$ Principal, ${ }^{3}$ Lecturer, ${ }^{4}$ Assistant Professor, ${ }^{1}$ Sagar Hospitals, Bengaluru, Karnataka, ${ }^{2-4}$ Karnataka, KLES' \\ Institute of Nursing Sciences, Hubli, Karnataka, India \\ *Corresponding Author: Rekha S G \\ Email: 73rekha@gmail.com
}

\begin{abstract}
Cancer may be a disease that involves the abnormal cell growth which originates especially tissues and organs of the body and metastasizes to other parts of the body. Cancer are often a results of several factors, like nutrition, environment, and physical activity, also as genetics. There are various sorts of cancer counting on their origin and organ they affect. carcinoma is that the top cancer in women worldwide and is increasing particularly in developing countries where the bulk of cases are diagnosed in late stages. Objective: This meta-analysis was conducted to research the impact of carcinoma on the mental and physical health status of girls. Methodology: The PRISMA tips for reportage of systematic reviews and meta-analyses were followed. An electronic search was administered in several health-related databases from 2000 to December 2015 using relevant search terms. Full-text, peer-reviewed articles in English those which include mental and physical status in longitudinal studies were considered for inclusion within the study. Results: a complete of 38 studies were included. Relevant data was gathered from 7896 samples with a mean age of fifty.71 years. Baseline assessment was done after diagnosis/surgery within the majority of the studies $(n=32,84.21 \%)$. The baseline assessment was done before diagnosis and after the diagnosis (29\%). the bulk of studies focused on just one indicator of psychological state ( $\mathrm{n}=28,73.68 \%$ ). Very commonly investigated studies were wont to assess mental and physical health status to carcinoma. Quality of Life, assessed in $(\mathrm{n}=15)$ studies, was the foremost common psychological outcome and was measured most frequently with the planet Health Organization Quality of Life. The second most ordinarily measured outcome was psychological distress $(\mathrm{n}=11)$. Results revealed that disease severity with metastatic disease were (43\%), patients with nonmetastatic disease $(27 \%)$, the remaining (30\%) didn't specify metastatic status. The (45\%) included patients undergoing or getting to begin cancer treatment (e.g., chemotherapy and/or radiotherapy), (52\%) patients either on or off treatment, and one (14\%) patients who had completed treatment.14 studies (36.84\%) were assessed at one assessment at short-term intervention and therefore the 24 remaining studies $(63.15 \%)$ were assessed at long-term duration. Low self-esteem (77\%), false self-perception (81\%), social isolation $(68 \%)$, negative body image $(45 \%)$, fatigue( $92 \%)$, relationship problems with family and friends $(74 \%)$, cancer stigma(39\%), fear of death (83\%), anxiety (78\%), loss of sexual desire(34\%), suicidal thought (23\%) and depression $(55.3 \%)$. $(\mathrm{N}=21,55.3 \%)$ of the patients experienced higher level of distress from alopecia. Conclusion: The management of carcinoma are often devastating and may produce to many adverse reactions for the bulk of girls. many ladies exhibit various symptoms of psychological distress like anxiety, depression, fatigue, pain, difficulty concentrating, social isolation, self-blame, spread of disease and fear of death etc. they'll have many doubts and questions associated with treatment protocol, side effects, precautions, duration of treatment, prognosis, Thus, healthcare professionals working with carcinoma patients should take initiative to know these concerns and support them by suggesting coping strategies that might help to alleviate their stress and anxiety.
\end{abstract}

Keywords: Breast cancer, Psychological state status, Physical health status, Psychological distress.

\section{Introduction}

Cancer is a disease that involves the abnormal cell growth which originates in particular tissues and organs of the body and metastasizes to other parts of the body. There are many contributing factors which could cause cancer, namely nutrition, environment, and physical activity, as well as genetics. There are various types of cancer depending on their origin and organ they affect. ${ }^{1}$

Breast cancer is one the very common cancer in women worldwide and which is increasing mainly in developing countries where the majority of cases are diagnosed in late stages. Breast Cancer is the most commonly diagnosed cancer among women, 1 in 8 
women. ${ }^{3}$ There are about 1.38 million new cases and 458000 deaths from breast cancer each year (IARC Globocan, 2008). Breast cancer is one of the most common cancer in women worldwide, In both the developing and under developed countries, has been rising up steadily in the few years due to rise in life expectancy, increase urbanization and adoption of western lifestyles. ${ }^{2}$

Incidence and mortality rate of cancer revealed by the American Cancer Society and other resources estimated annual incidence for 2019 had to be 40,000 cases or more. The most common type of cancer is breast cancer, with 271,270 new cases expected in the United States in 2019. ${ }^{3}$

Causes of cancer are many like; tobacco use- $22 \%$, life style $-10 \%$, obesity, poor diet, lack of physical activity, excessive drinking of alcohol, infections$15 \%$, helicobacter pylori, hepatitis $\mathrm{B}$, hepatitis $\mathrm{C}$, human papillomavirus infection, Epstein- Barr virus, $\mathrm{HIV}$, exposure to ionizing radiation and environmental pollutants, changing gene of a cell, inherited genetic factors-5-10\%. Greater than 70-90\% of common cancers are due to environmental factors and which can be prevented by modifying our life styles - quit smoking, maintain healthy weight, not drinking too much alcohol, eating plenty of vegetables, fruits and whole grains, vaccination, avoiding processed and red meat, avoiding exposure to sunlight, early detection and prompt treatment. ${ }^{1,4,5}$

Statistical findings mentioned approximately 18 million cancer cases around the world in 2018, of these 9.5 million cases were in men and 8.5 million in women. With the growing global burden, prevention of cancer is one of the most significant public health challenges of the 21st century. Global cancer incidence in each sexes; respiratory organ and breast cancers were the foremost common cancers worldwide, each contributing $12.3 \%$ of the total number of new cases diagnosed in 2018. Colorectal cancer was the third most typical cancer with one. 8 million new cases in 2018. Breast cancer was the foremost common cancer in ladies worldwide, contributing $25.4 \%$ of the total number of new cases diagnosed in 2018. The top 3 - breast, colorectal and lung cancers - contributed $43.9 \%$ of all cancers (excluding non-melanoma skin cancer). Cervical cancer was the fourth most common cancer in women, contributing $6.9 \%$ of the total number of new cases diagnosed in $2018 .^{5}$

\section{Background}

Cancer may be a cluster of sickness involving abnormal cell growth with the potential to invade to different components of the body. Cancer is that the second leading explanation for death within the world behind upset. ${ }^{3}$ It is calculable that each sixth death within the world is thanks to cancer. While the entire variety of deaths from cancer is increasing, this is likely the result of aging and growing populations. Luckily, cancer treatment advancements across the spectrum of sorts of cancers ar continued at a fast pace. Many people who are diagnosed with cancer are living longer or are cured. ${ }^{1}$

The data revealed that Australia has the highest cancer rate in the world of 468 cases per 100,000 people. Australia specifically has a number of the very best rates of carcinoma and malignant melanoma within the world. Syria has the bottom cancer rate within the world of eighty five cases per a hundred, 000 people. Bhutan, Algeria, Nepal, and Oman followed with rates below $100 .^{3}$

Two reports released on the same day say that cancer is the second biggest killer in India, $8.17 \%$ of cancer deaths in the world this year. Cancer will claim 9.6 million lives in the world this year and India's share in it will be a $8.17 \%$, data reported by the World Health Organization's (WHO) International Agency for Research on Cancer. Another report free at intervals a niche of few hours by Lancet bolstered the gloomy prediction concerning the sickness spoken language it's India's second biggest killer when heart condition.

The United Nations agency information says Asian country can have one.16 million new cancer cases this year and more than 50 per cent of these will be diagnosed in women. Or, exactly seventeen,204 more women will fall prey to the disease than men. Moreover, breast cancer incidence has gone up by $39.1 \%$ between 1990 and 2016 and is the most common cancer among women in India, accounting for the largest crude incidence rate and prevalence of any cancer type, says the Lancet report. In India, we 
tend to ar currently witnessing a lot of and a lot of numbers of patients being diagnosed with carcinoma to be within the younger age teams.

Cancer is the second leading cause of death in the world after cardiovascular disease. It is estimated that every sixth death in the world is due to cancer. While the total number of deaths from cancer is increasing, this is likely the result of aging and growing populations. The lack of awareness, suboptimal medical infrastructure, less availability of screening, and low doctor-patient ratio is the prime. More than $50 \%$ of the cases in India are diagnosed in stage 3 or 4, which decreases the patients' chances of survival. Reports say Asian country has highest mortality-toincidence magnitude relation within the whole world. There is a desire for a holistic approach towards cancer treatment in Asian country. We need a well-planned treatment pathway - from bar to early detection and complete treatment, aside from affordability and accessibility.

Psychological stress is feeling when we are under mental, physical, or emotional pressure. Although it's traditional to expertise some psychological stress from time to time, people who experience high levels of psychological stress or who experience it repeatedly over a long period of time may develop health problems (mental or physical). Stress is caused each by daily responsibilities and routine events, as well as by more unusual events, such as a trauma or illness in oneself or a close family member. When folks feel that they're unable to manage or management changes caused by cancer or traditional life activities, they are in distress. Distress has become progressively recognized as an element that may scale back the standard of lifetime of cancer patients. There is even some evidence that extreme distress is associated with poorer clinical outcomes, the prognosis of cancer can range from excellent to poor, it also depends on the type of cancer and stage of cancer. People who have cancer might notice the physical, emotional, and social effects of the disease to be stressful. Those World Health Organization commit to manage their stress with risky behaviours like smoking or drinking alcohol or who become additional inactive might have a poorer quality of life when cancer treatment. In distinction, people who are able to use effective coping strategies to deal with stress, such as relaxation and stress management techniques, have been shown to have lower levels of depression, anxiety, and symptoms related to the cancer and its treatment. ${ }^{6}$

During the process of cancer treatment, one may have hair loss, experience weight changes, get surgical scars or have body parts surgically altered/removed. It is normal for these events to change a person's sex drive and body image. In women, the loss of the symbols of femininity can result in low self-esteem, negative body image, false self-perception, social isolation and the development of communication or relationship problems with family members or friends. ${ }^{7,8}$ As a result of the side effects of cancer treatments, some women may develop "cancer stigma" from losing their feminine physical characteristics through hair loss or the loss of one or both breasts.

Depression is a really necessary mental issue for cancer patients. It is calculable that $16-25 \%$ of cancer patients develop depression. Doctors don't acknowledge concerning thirty fifth of those cases and lots of patients stay untreated. Depression is additionally additional common in cancer patients than the final population. There square measure many classes of depression with major depression being the foremost noticeable sort.

\section{Objective}

This meta-analysis was conducted to investigate the impact of breast cancer on the mental and physical health status of women.

\section{Methodology}

The PRISMA tips for reportage of systematic reviews and meta-analyses were followed. An electronic search was carried out in different health-related databases from 2000 to December 2015 using relevant search terms. Full-text, peer-reviewed articles in English those which include mental and physical status in longitudinal studies were considered for inclusion in the study.

\section{Literature search criteria}

Investigator conducted a systematic search of publicly available peer-reviewed articles and reports from April 1, 2005 to March 31, 2018. Key words used for search 
were a. breast cancer, mastectomy b. mental health status, psychological adjustment, stress, adaptation, psychological issues, depression, anxiety, emotional distress, emotional adjustment, quality of life, physical health, body image disturbances, c. longitudinal study or prospective study. Academic Search Complete, WedMD CINAHL plus, Science direct, ERIC, NHS Direct, MedicLatina, MEDLINE, PsycCRITIQUES, PsycARTICLES, PsycINFO, Psychology and Behavioral Sciences Collection. Apart from the above we also supplemented the search by additional handsearching in Google and checking reference lists were used to identify any additional publications that may have been missed

\section{Eligibility criteria Inclusion criteria}

Chosen inclusion criteria for the selected study were studies which were aimed to analyse the impact of breast cancer on mental and physical health status.

\section{Inclusion criteria}

1. Only Quantitative and longitudinal studies

2. Independent variables including demographics, disease related variables

3. Mental and physical status at the time of diagnosis, after the diagnosis, during the treatment and during the follow ups

4. Outcome variables were expression of mental adjustment throughout the disease progression.

\section{Exclusion criteria}

1. Studies related to other type of cancer

2. Unpublished studies, abstracts of conferences, case reports, and qualitative studies, books

3. Cross sectional studies

4. Study sample size less than 25

5. No restrictions on the number of mental and physical health assessments, follow up duration

\section{Data analysis}

Two authors reviewed all reports and entered information from those meeting initial inclusion criteria into an proof table custom-made from PRISMA statement parts. We categorised information supported geographical region wherever the study/evaluation was conducted, year, institution/organization that carried it out, its objectives, its design and methods. We known the kind of study/evaluation they utilized organized them into four broad classes. Two authors screened the titles and abstracts which meet the inclusion criteria were included for meta-analysis. Independent inter- rater scoring was given to each selected article by the two authors. No discrepancies in inclusion decisions between reviewers were found.

\section{Results}

In the beginning there was a pool of 280 articles. A total of 38 studies were included. Studies were conducted in the different parts the world, most commonly USA, United Kingdom, Africa, Netherlands, Australia, Canada, France, Greece, Hong Kong, Iran, Japan, Norway, Portugal, Oman, Spain, South Korea, Sweden, Taiwan, and India. Relevant data was gathered from 7896 samples with a mean age of 50.71 years. The number of mental and physical assessment duration in these studies ranged from two to six weeks and follow-up duration was varied from two months post-diagnosis to ten years postdiagnosis. Baseline assessment was done after diagnosis/surgery in the majority of the studies $(n=32,84.21 \%)$. The baseline assessment was done before diagnosis and after the diagnosis (29\%). The majority of studies focused on only one indicator of mental health $(n=28$, $73.68 \%)$.

Very commonly investigated studies were used to assess mental and physical health status to breast cancer. Quality of Life, assessed in $(n=15)$ studies, was the most common psychological outcome and was measured most often with the World Health Organization Quality of Life. The second most commonly measured outcome was psychological distress $\quad(n=11) . \quad$ Self-constructed structured instruments were used to assess psychological distress. The most common were the General Health Questionnaire (GHQ), the Hospital Anxiety and Depression Scale (HADS), and the Profile of Mood State (POMS). Depressive symptoms were the third most commonly measured outcome with the Center for Epidemiologic Studies Depression Scale being the most common instrument used $(\mathrm{n}=6)$. Anxiety 
symptoms were evaluated by four studies, mainly with the HADS. The least oft measured psychological outcome was mood, which was assessed by two studies using the POMS or the PANAS.

The items were organized into three categories: Demographic variables, disease related variables and mental and physical health variables. Results revealed that disease severity with metastatic disease were $(43 \%)$, patients with nonmetastatic disease $(27 \%)$, the remaining (30\%) did not specify metastatic status. The $(45 \%)$ included patients undergoing or going to begin cancer treatment (e.g., chemotherapy and/or radiotherapy), (52\%) patients either on or off treatment, and one (14\%) patients who had completed treatment.

14 studies (36.84\%) were assessed at a single assessment at short-term intervention and the 24 remaining studies $(63.15 \%)$ were assessed at long-term duration.

Among these 38 studies, women experienced variety of symptoms exhibiting the low mental and health status. Low self-esteem (77\%), false selfperception $(81 \%)$, social isolation(68\%), negative body image $(45 \%)$, fatigue( $92 \%)$, relationship problems with family and friends(74\%), cancer stigma(39\%), fear of death(83\%), anxiety(78\%), loss of sexual desire(34\%), suicidal thought(23\%) and depression(55.3\%). $(\mathrm{N}=21,55.3 \%)$ of the patients experienced higher level of distress from alopecia.

Results shown carcinoma patients just about forty second older GAD, an anxiety disorder in which a general feeling of unease or fear is present, despite little or no threat. GAD sufferers pay most of the day worrying, often to the point of mental exhaustion, and experience physical symptoms such as headaches, irritability and trembling.

\section{Discussion}

Researchers have found that girls World Health Organization square measure diagnosed with carcinoma oft expertise depressive symptoms that impact their quality of life moreover as adherence to treatment. Some of the conditions that a breast cancer patient may experience include; Severe Emotional Distress, Major Depression, Post-Traumatic Stress
Disorder (PTSD), Generalized Anxiety Disorder (GAD). Symptoms experienced by women vary; Change in appearance, general unhappiness, negative thoughts: continuous feeling of worthlessness to others, hopelessness about future, reduced activity, reduced concentration, people problems, guilt and low self-esteem, physical problems, suicidal thoughts, reliving the moment, avoidance, increased arousal. Body image has been named because the image of our own body that we have a tendency to type in our mind, and associated characteristics include expression of emotions, imitation, identification, beauty, and social aspects.

\section{Conclusions}

Cancer cases and mortality are increasing among young women, primarily due to low awareness and late detection. India accounts for the third highest range of cancer cases among girls once China and therefore the North American country, and is growing annually at four.5-5 p.c, consistent with new information.

The management of breast cancer can be devastating and can give rise to several adverse reactions for the majority of women. Many women exhibit various symptoms of psychological distress such as anxiety, depression, fatigue, pain, difficulty concentrating, social isolation, self-blame, spread of disease and fear of death etc. They may have lots of doubts and questions related to treatment protocol, side effects, precautions, duration of treatment, prognosis, Thus, healthcare professionals working with breast cancer patients should take initiative to understand these concerns and support them by suggesting coping strategies that would help to relieve their stress and anxiety.

\section{Reference}

1. Wikipedia. https://en.wikipedia.org/wiki/Cancer

2. Breast Cancer Awareness Month in October https://www.who.int/cancer/events/breast_cancer_month/en /

3. Cancer Rates By Country 2019 http://worldpopulationreview.com/countries/cancer-ratesby-country/

4. American Cancer Society: Cancer Facts and Figures 2019. Atlanta, Ga: American Cancer Society, 2019. Also 
available online Exit Disclaimer. Last accessed February 5, 2019.

5. Bray F, Ferlay J, Soerjomataram I, Siegel RL, Torre LA, Jemal A. Global Cancer Statistics 2018: GLOBOCAN estimates of incidence and mortality worldwide for 36 cancers in 185 countries. CA Cancer J Clin, in press. The online GLOBOCAN 2018 database is accessible at http://gco.iarc.fr/, as part of IARC's Global Cancer Observatory.https://www.wcrf.org/dietandcancer/cancertrends/worldwide-cancer-data

6. Cancer Breast cancer Lancet The World Health International Agency Health India https://www.downtoearth.org.in/news/health/9-6-millionpeople-will-die-of-cancer-this- year-61646

7. Cancer Epidemiology, Biomarkers \& Prevention, April 2004

Volume 13, Issue 4; https://cebp.aacrjournals.org/content/13/4/501.full

8. Avis NE, Levine BJ, Case LD, Naftalis EZ, Van zee KJ. Trajectories of depressive symptoms following breast cancer diagnosis. Cancer Epidemiol Biomarkers Prev 2015;24(11):1789-95. doi:10.1158/1055-9965.EPI-15-0327

9. National Comprehensive Cancer Network. Managing Stress and Distress.

10. Patrick DL, Ferketich SL, Frame PS, Harris JJ, Hendricks CB, Levin B et al. National Institutes of Health State-ofthe-Science Panel. National Institutes of Health State-ofthe-Science Conference Statement: Symptom Management in Cancer: Pain, Depression, and Fatigue, (2002) J Natl Cancer Inst 2003;95(15):1110-7 [PUBMED]

11. Fisch MJ, Callahan CM, Kesterson JG.: The use of an electronic patient record system to identify advanced cancer patients and antidepressant drug use. J Palliat Med 1999;2(4):403-9. [PUBMED]

12. Bottomley A (1998) Depression in cancer patients: a literature review. Eur J Cancer Care 7(3):181191 [PUBMED]

13. Massie MJ. Prevalence of depression in patients with cancer. J Natl Cancer Inst Monogr 2004;(32):57-71 [PUBMED]

14. BreastCancer.org. Depression. Updated April 17, 2019.

15. Voigt V, Neufeld F, Kaste J. Clinically assessed posttraumatic stress in patients with breast cancer during the first year after diagnosis in the prospective, longitudinal, controlled COGNICARES study. Psychooncology 2017;26(1):74-80. doi:10.1002/pon.4102

16. Tsaras K, Papathanasiou IV, Mitsi D, et al. Assessment of Depression and Anxiety in Breast Cancer Patients: Prevalence and Associated Factors. Asian Pac J Cancer Prev 2018;19(6):1661-9. doi:10.22034/APJCP.2018.19.6.1661

17. Artherholt SB, Fann JR. Psychosocial care in cancer. Curr Psychiatry Rep 2012;14(1):23-29. [PubMed Abstract]

18. Fashoyin-Aje LA, Martinez KA, Dy SM. New patientcentered care standards from the Commission on Cancer: opportunities and challenges. J Supportive Oncol 2012; epub ahead of print March 20, 2012. [PubMed Abstract]

19. Lutgendorf SK, DeGeest K, Dahmoush L. Social isolation is associated with elevated tumor norepinephrine in ovarian carcinoma patients. Brain, Behavior, Immunity 2011;25(2):250-5. [PubMed Abstract]

20. Lutgendorf SK, Sood AK, Anderson B. Social support, psychological distress, and natural killer cell activity in ovarian cancer. J Clin Oncol 2005;23(28):7105-

13. [PubMed Abstract]

21. Lutgendorf SK, Sood AK, Antoni MH. Host factors and cancer progression: biobehavioral signaling pathways and interventions. J Clin Oncol 2010;28(26):4094-9. [PubMed Abstract]

22. McDonald PG, Antoni MH, Lutgendorf SK. A biobehavioral perspective of tumor biology. Discov Med 2005;5(30):520-26.[PubMed Abstract]

23. Melhem-Bertrandt A, Chavez-Macgregor M, Lei X. Betablocker use is associated with improved relapse-free survival in patients with triple-negative breast cancer. $J$ Clin Oncol 2011;29(19):2645-52. [PubMed Abstract]

24. Moreno-Smith M, Lutgendorf SK, Sood AK. Impact of stress on cancer metastasis. Future Oncol 2010;6(12):186381. [PubMed Abstract]

25. Segerstrom SC, Miller GE. Psychological stress and the human immune system: a meta-analytic study of 30 years of inquiry. Psychological Bull 2004;130(4):601630. [PubMed Abstract]

26. Sloan EK, Priceman SJ, Cox BF. The sympathetic nervous system induces a metastatic switch in primary breast cancer. Cancer Res 2010;70(18):7042-7052. [PubMed Abstract]

27. https://www.pennmedicine.org/cancer/about/focus-oncancer/2012/october/10-mustknow-acts-about-breastcancer

28. https://www.cancer.org/cancer/breast-cancer/about/howcommon-is-breast-cancer.html

29. https://www.bcrf.org/breast-cancer-statistics-and-resources

30. https://www.roche.com/research_and_development/what_ we_are_working_on/oncology/8-facts-about-breastcancer.htm

31. U.S.Breast Cancer Statistics; https://www.breastcancer.org/symptoms/understand_bc/stat istics

32. http://local.psy.miami.edu/faculty/ccarver/pHPConcerns.pd $\mathrm{f}$

33. Collaborative Group on Hormonal Factors in Breast Cancer. Menarche, menopause, and breast cancer risk: individual participant meta-analysis, including 118,964 women with breast cancer from 117 epidemiological studies. Lancet Oncol 2012;13:1141-51

34. Shi R, Taylor H, McLarty J, Liu L, Mills G, Burton G et al. Effects of payer status on breast cancer survival: a retrospective study. BMC Cancer 2015;15:211.

35. White MC, Espey DK, Swan J, Wiggins CL, Eheman C, Kaur JS et al. Disparities in cancer mortality and incidence 
among American Indians and Alaska Natives in the United States. Amer J Pub Health 2014;104(3): S377-87.

36. Mindfulness-based stress reduction and cancer: a metaanalysis

https://onlinelibrary.wiley.com/doi/pdf/10.1002/pon.1400\# accessDenialLayout

37. Haque R, Ahmed SA, Inzhakova G. Impact of breast cancer subtypes and treatment on survival: an analysis spanning two decades. Cancer Epidemiol Biomarkers Prev 2012;21:1848-55

38. "Cancer - Signs and symptoms". NHS Choices. Arch Original on 8 June 2014. Retrieved 10 June 2014.

39. Cancer. World Health Organization. 12 September 2018. Retrieved 19 December 2018.

40. https://onlinelibrary.wiley.com/doi/pdf/10.1002/pon.1400\# accessDenialLayout
41. http://worldpopulationreview.com/countries/cancer-ratesby-country/

42. Anand P, Kunnumakkara AB, Sundaram C, Harikumar $\mathrm{KB}$, Tharakan ST, Lai OS et al,. "Cancer is a preventable disease that requires major lifestyle changes". Pharm Res 2008;25(9):2097-116. doi:10.1007/s11095-008-96619. PMC 2515569. PMID 18626751.

43. National Cancer Institute (26 February 2018). "Targeted Cancer Therapies"

How to cite this article: Rekha SG, Peerapur SM, Roy A, Tejaswini BH. Cancer is a word, not what defines you: Women with breast cancer. J PaediatrNurs Sci 2019;2(4):85-91. 